\title{
Regulation of Behavioral and Synaptic Plasticity by Serotonin Release within Local Modulatory Fields in the CNS of Aplysia
}

\author{
Stéphane Marinesco, ${ }^{1,2}$ Nimalee Wickremasinghe, ${ }^{1}$ and Thomas J. Carew ${ }^{1}$ \\ ${ }^{1}$ Department of Neurobiology and Behavior, Center for the Neurobiology of Learning and Memory, University of California, Irvine, California 92697-4550, \\ and ${ }^{2}$ Institut de Neurobiologie Alfred Fessard, Fédération de Recherche du Centre National de la Recherche Scientifique (CNRS) 2118, Laboratoire de \\ Neurobiologie Cellulaire et Moléculaire, Unité Propre de Recherche 9040, CNRS, Gif sur Yvette F-91198, France
}

In Aplysia, serotonergic neurons are widely activated during sensitization training, but the effects of exogenous serotonin (5-HT) on reflex circuits vary, inducing short- or long-term synaptic facilitation or synaptic inhibition, depending on the site of application. During learning, it is possible that specific spatial patterns of 5-HT release evoked by training may produce different phases of sensitization or behavioral inhibition. To test this hypothesis, we examined the modulation of the tail-induced siphon withdrawal reflex by repeated noxious stimuli applied to one of three sites: the (1) ipsilateral or (2) contralateral sides of the tail or (3) the head. Ipsilateral tail shock produced long-term sensitization, whereas contralateral tail shock induced only short-term sensitization, and head shock produced inhibition. In parallel cellular experiments, tail-nerve shock evoked large 5-HT release localized around the ipsilateral tail sensory neurons (SNs) and motor neurons (MNs) but only modest 5-HT release in the contralateral pleural-pedal ganglia and in the abdominal ganglion, in which the siphon MNs are located. Head-nerve shock, in contrast, produced only modest 5-HT release in the pleural, pedal, and abdominal ganglia. Thus, each training protocol evoked a specific pattern of 5-HT release within the CNS. In addition, we found that 5-HT released in the pleural ganglia was correlated with facilitation of SN-MN synapses; however, in the abdominal ganglion, it was associated with inhibition of the synapses between identified interneurons (L29s) and siphon MNs (LFSs). Because 5-HT differentially modulates synaptic efficacy at different synaptic sites, our data can explain how specific spatial patterns of 5-HT release in local modulatory fields can contribute to the induction of short- or long-term sensitization or to behavioral inhibition.

Key words: invertebrate; memory; sensitization; 5-hydroxytryptamine; neuromodulation; amperometry

\section{Introduction}

Serotonin $(5-\mathrm{HT})$ is a highly conserved neurotransmitter that modulates complex behaviors across a wide range of species. Understanding the mechanisms of 5-HT modulation in any given neuronal network has often proved difficult because of the widespread projections of serotonergic neurons and the multiplicity of 5-HT receptors. The marine mollusk Aplysia is a useful model system to address this general question, because it allows the study of identified neural circuits at the behavioral, cellular, and molecular levels (Kandel, 1979). In addition, the serotonergic system of Aplysia presents many homologies with mammalian species. For example, serotonergic neurons in Aplysia are involved in arousal, feeding, and locomotion (Kupfermann and Weiss, 1982; Mackey and Carew, 1983; Lloyd et al., 1984; Rosen et al., 1989; McPherson and Blankenship, 1992; Marinesco et al., 2004b), like their counterparts in mammals (Veasey et al., 1995, 1997) ( for review, see Jacobs and Fornal, 1999; Meguid et al., 2000; Siegel, 2004). Moreover, as in the mammalian CNS, sero-

Received Aug. 1, 2006; revised 0ct. 2, 2006; accepted 0ct. 27, 2006.

This work was supported by National Institutes of Health Grant R01 MH14-10183 (T.J.C.). We are grateful to

Carolyn Sherff and Gary Philips for helpful comments on a previous version of this manuscript.

Correspondence should be addressed to Thomas J. Carew at the above address. E-mail: tcarew@uci.edu.

D01:10.1523/JNEUROSCI.3309-06.2006

Copyright $\odot 2006$ Society for Neuroscience $\quad$ 0270-6474/06/2612682-12\$15.00/0 tonergic neurons in Aplysia are tonically active at rest, fire at relatively low frequencies (usually $<1 \mathrm{~Hz}$ ) (Kupfermann and Weiss, 1982; Marinesco et al., 2004a), and can release 5-HT in a paracrine or volumic manner (Marinesco and Carew, 2002a; Zhang et al., 2003) (for review, see Bunin and Wightman, 1999).

One of the most studied forms of serotonergic modulation in Aplysia is sensitization of defensive reflexes after noxious stimulation of the tail (Barbas et al., 2003) (for review, see Byrne and Kandel, 1996). 5-HT is necessary for the induction of sensitization (Glanzman et al., 1989), and tail shock increases the firing rate of a large number of serotonergic neurons (Mackey et al., 1989; Marinesco et al., 2004a). Moreover, 5-HT is released within the CNS (Marinesco and Carew, 2002a) and hemolymph (Levenson et al., 1999) in response to noxious stimuli. At the cellular level, 5-HT can modulate synaptic strength in several different ways. It facilitates sensory neuron (SN)-motor neuron (MN) connections through activation of a $\mathrm{G}_{\mathrm{s}}$-coupled receptor (Brunelli et al., 1976; Mercer et al., 1991; Byrne and Kandel, 1996; Barbas et al., 2003; Cohen et al., 2003), but also exerts inhibitory actions at several other synapses (Frost et al., 1988; Fischer and Carew, 1993; Xu et al., 1995; Storozhuk and Castellucci, 1999; Bristol et al., 2001). Thus, in the tail-induced siphon withdrawal (T-SW) reflex, 5-HT can contribute to both sensitization, by facilitating synaptic transmission from tail SNs, and reflex inhibition, by inhibiting the excitatory synapses from identified inter- 
A. Head vs. Tail shock
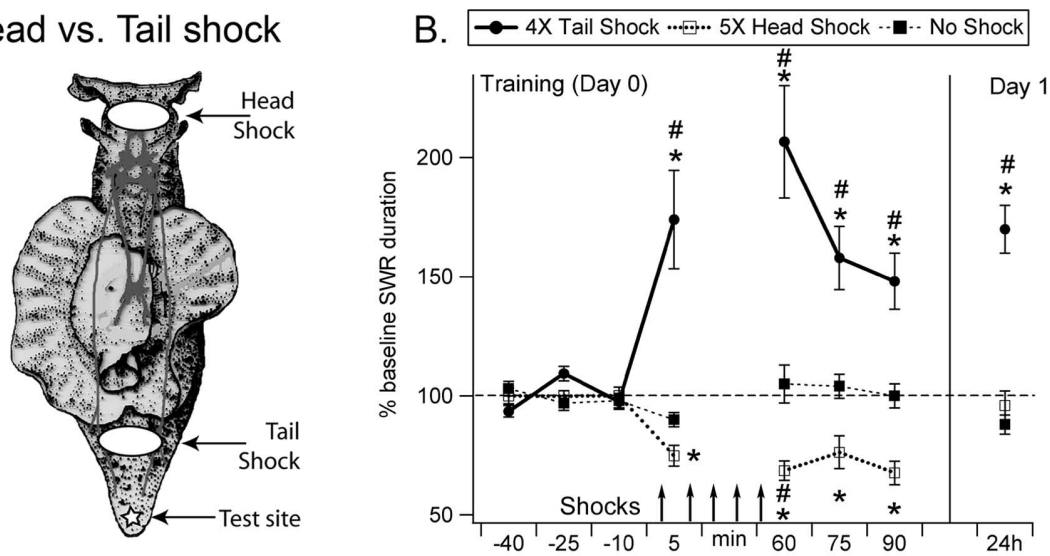

C. Ipsi vs. Contralateral shock D.
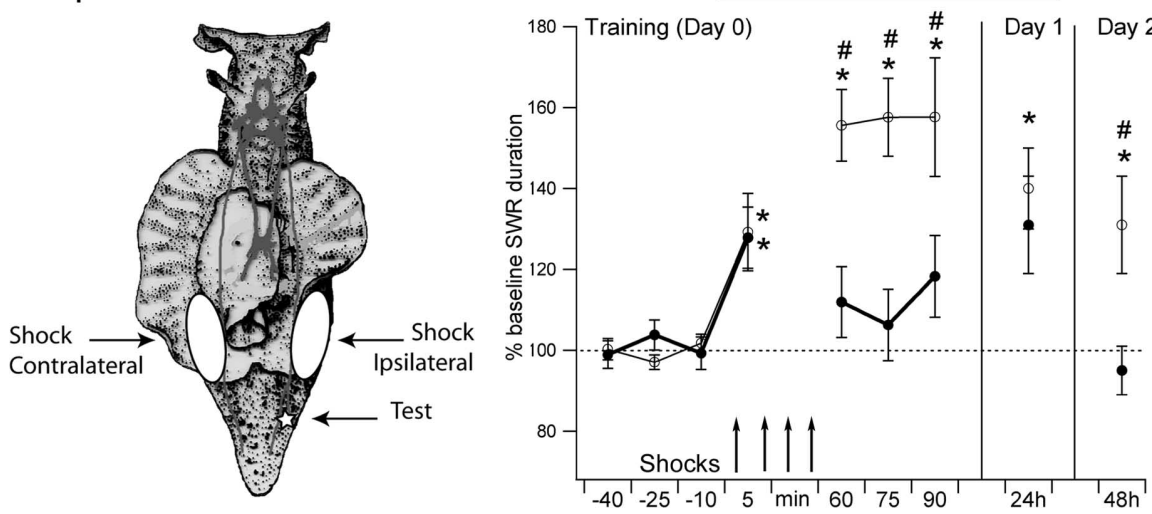

Figure 1. T-SW reflex modulation after electrical shocks applied to the tail or to the head. $\boldsymbol{A}$, Localization of head and tail shocks and of the site of testing on the tail. $\boldsymbol{B}$, Effects of tail versus head shock on T-SW reflex. Significant short-, intermediate-, and long-term sensitization was exhibited after four tail shocks (ISI, $15 \mathrm{~min}$ ). In contrast, the reflex was inhibited for at least $1.5 \mathrm{~h}$ after five shocks applied to the head and returned to baseline levels $24 \mathrm{~h}$ after training. ${ }^{*}$ Significantly different from baseline; " significantly different from no-shock group ( 4 tail shocks, $n=16 ; 5$ head shocks, $n=11$; no shock, $n=15$ ). C, Localization of ipsilateral (Ipsi) and contralateral tail shock and of the test site. D, Effects of tail shock on the ipsilateral or contralateral side of the tail. On the ipsilateral side, significant short-, intermediate-, and long-term sensitization was exhibited, whereas the contralateral T-SW reflex only showed short-term sensitization. *Significantly different from baseline; " significantly different from contralateral group (ipsilateral, $n=15$; contralateral, $n=10$ ). Error bars respresent SEM. SWR, Siphon withdrawal reflex.

neurons (the L29s) to siphon MNs (the LFS neurons) (Bristol et al., 2001).

In this study, we sought to understand how these different 5-HT modulatory effects are selected during learning. First, we found that the T-SW reflex can be bidirectionally modulated after noxious stimuli: it is sensitized for $48 \mathrm{~h}$ after ipsilateral tail shock, sensitized for only 15-30 $\mathrm{min}$ in response to contralateral tail shock, and inhibited in response to head shock. Although all three of these noxious stimuli increase the firing rate of serotonergic neurons and trigger the release of 5-HT in the CNS, the spatial pattern of the evoked 5-HT release is different in each case. Next, using the high temporal and spatial resolution of chronoamperometric techniques, we were able to identify steep gradients in 5-HT extracellular concentrations that define local hot spots of maximal 5-HT release. Thus, in this reflex circuit, independent regulation of 5-HT release within restricted modulatory fields allows the CNS to differentiate between different serotonergic release patterns that, in turn, can give rise to both long-term and transient reflex sensitization, as well as to reflex inhibition.

\section{Materials and Methods}

Animals and preparation of isolated ganglia. Wild-caught adult Aplysia californica [Marinus (Long Beach, CA) or MREP (Oceanside, CA)] weighing $\sim 250$ g were used throughout this study. Animals were housed in tanks containing aerated artificial seawater (ASW) kept at $15^{\circ} \mathrm{C}$ under a $12 \mathrm{~h}$ light/dark cycle. To prepare the ganglia, animals were first anesthetized by injection of $150-200 \mathrm{ml}$ of $0.35 \mathrm{M} \mathrm{MgCl}_{2}$ and immediately dissected for removal of the entire CNS except the buccal ganglion. The ganglia were then fixed in $0.4 \%$ glutaraldehyde for $\sim 30$ $s$ and desheathed in 50:50 ASW: $0.35 \mathrm{M} \mathrm{MgCl}_{2}$ to expose the neurons. Ganglia were then kept in a recording chamber at room temperature, continuously perfused with ASW [(in mM) 460 $\mathrm{NaCl}, 55 \mathrm{MgCl}_{2}, 11 \mathrm{CaCl}_{2}, 10 \mathrm{KCl}$, and 10 Tris]. ASW was buffered to $\mathrm{pH} 7.6$ before use.

Behavioral studies. Behavioral experiments were performed in the home tanks at $15^{\circ} \mathrm{C}$. Parapodia were surgically removed to directly visualize siphon contractions. Animals were anesthetized in ice-cold ASW for $1 \mathrm{~h}$ before surgery and left undisturbed for at least $5 \mathrm{~d}$ before behavioral experiments. The siphon withdrawal reflex was evoked by a mild tactile stimulus applied to the tip of the tail with a water jet (Teledyne Waterpik, Fort Collins, CO). The T-SW reflex was induced at a 15 min interstimulus interval (ISI), and the duration of the siphon withdrawal was measured between water jet onset and the first signs of siphon relaxation (Sutton et al., 2002). For behavioral training, animals were placed in a shock box in which they received electrical shocks and were allowed to ink for $5-10 \mathrm{~s}$ before returning into their home tank for testing. Electrical shocks were applied to the head [between anterior tentacles and posterior rhinophores (Fig. 1A)] or to the tail (Fig. $1 A, C$ ) with a suction electrode, using five $2 \mathrm{~s}$ trains of current pulses spaced by $5 \mathrm{~s}$ (current pulses were $10 \mathrm{~ms}$ at $30 \mathrm{~mA}$, applied at $50 \mathrm{~Hz}$ through a constant-current stimulus isolation unit connected with an S88 stimulator; Grass Medical Instruments, Quincy, MA) ( $5 \mathrm{~s}$ interval between the beginning of two consecutive pulses). We always applied the noxious training stimuli at a different site from the one used for testing memory. In particular, when we examined the effects of ipsilateral tail shock, electrical shocks were applied near the base of the parapodia, whereas tests were performed near the tip of the tail (Fig. 1C). Thus, we could exclude the possibility that parts of the skin stimulated when testing had been injured during training. For control experiments, a group of animals was placed in the shock box, and the suction electrode was applied to their skin, but no current was passed. This procedure produced no significant effect on the T-SW reflex duration (Fig. $1 B$ ). Tail shock was applied to the right or left side of the animal at random, and the reflex was tested either ipsilateral or contralateral to the shock.

Previous studies from our laboratory have determined that four tail shocks spaced by $15 \mathrm{~min}$ is a minimal training required for the induction of robust long-term sensitization (Sutton et al., 2002). For head shock, we found that five shocks spaced by 15 min were more effective in inducing behavioral inhibition. When we tested four spaced head shocks, behavioral inhibition measured 15-45 min after the end of training was less pronounced $(-15 \pm 5 \% ; p<0.01)$ than after five head shocks $(-29 \pm$ $5 \% ; p<0.01$ ) (Fig. 1B). Throughout the study, behavioral data are presented as short-term memory (T-SW reflex duration measured 5 min after the first shock), intermediate-term memory (average of three tests 15,30 , and $45 \mathrm{~min}$ after the last shock), and long-term memory (average of three tests spaced $15 \mathrm{~min}$ apart 24 or $48 \mathrm{~h}$ after training) (Sutton et al., 2002). T-SW reflex duration was expressed as the percentage of the base- 
line response (average of three pretests 10, 25, and $40 \mathrm{~min}$ before training).

Chronoamperometry. Chronoamperometric detection of 5-HT was achieved as described previously by Marinesco and Carew (2002a,b). In the isolated CNS, carbon fiber electrodes $(7 \mu \mathrm{m}$ diameter; $300 \mu \mathrm{m}$ length) were implanted in the neuropil of abdominal, pedal, or pleural ganglia. Chronoamperometric pulses consisted of four successive voltage steps ( $80 \mathrm{mV}$ for $40 \mathrm{~ms}, 230 \mathrm{mV}$ for $15 \mathrm{~ms}, 250 \mathrm{mV}$ for $40 \mathrm{~ms}$, and $400 \mathrm{mV}$ for $15 \mathrm{~ms}$ ) applied between the working and reference electrodes at $2 \mathrm{~Hz}$. Oxidation currents were measured using a VA10 voltammeter (NPI Electronic, Tamm, Germany) connected to a three-electrode potentiostat. Serotonin-related oxidation currents were measured as the difference between the currents measured at the end of the fourth pulse $(400 \mathrm{mV})$ and the third pulse $(250 \mathrm{mV})$. At the end of the recording, the carbon fiber electrode was removed from the ganglion and calibrated in a flow injection chamber with $500 \mathrm{~nm}$ 5-HT. The oxidation current obtained during the calibration was used to estimate the actual 5-HT concentration in the ganglion.

Staining of serotonergic neurons and immunohistochemistry. In one experiment, we stained serotonergic neurons in living animals using 5,7dihydroxytryptamine (5,7-DHT) (Jahan-Parwar et al., 1987; Marinesco et al., 2004a). For this purpose, animals were injected twice with $10 \mathrm{mg} / \mathrm{kg}$ 5,7-DHT dissolved at $5 \mathrm{mg} / \mathrm{ml}$ in ASW, at a 1 week interval. Drugs were administered by intrahemocoel injections through the foot. Animals were then left undisturbed for 2 months before experimentation. In these animals, serotonergic cells developed a dark pigmentation that can be observed in living ganglia under a regular light microscope. To confirm that these cells were indeed serotonergic, we stained isolated ganglia using an antibody against 5-HT as described previously (Marinesco et al., 2004a): the ganglia were fixed overnight with $4 \%$ formaldehyde at $4^{\circ} \mathrm{C}$ and permeabilized with $4 \%$ Triton X-100 (Sigma, St. Louis, MO) in PBS for $1 \mathrm{~h}$ at room temperature. Nonspecific binding was blocked by immersion in $10 \%$ goat serum (Jackson ImmunoResearch, West Grove, $\mathrm{PA}$ ) with $0.5 \%$ Triton $\mathrm{X}-100$ for $1 \mathrm{~h}$ before exposure to the rabbit polyclonal anti-serotonin antibody (Immunostar, Hudson, WI) at 1:1000 for $1.5-2.5 \mathrm{~d}$ at $4^{\circ} \mathrm{C}$. Visualization of the primary antibody to 5 -HT was performed with a goat anti-rabbit secondary antibody coupled to cyanine 5 (Cy5; dilution, $1: 100 ; 3 \mathrm{~h}$ at room temperature; Jackson ImmunoResearch).

After recording from neurons stained by 5,7-DHT, we injected them with Neurobiotin using ionophoresis for $45 \mathrm{~min}$ to $1 \mathrm{~h}$ ( $+5 \mathrm{nA}$ pulses; $500 \mathrm{~ms}$ duration at $1 \mathrm{~Hz}$ ), to subsequently identify them using confocal microscopy. Neurobiotin was then revealed using streptavidin coupled to Alexa Fluor $488\left(10 \mu \mathrm{g} / \mathrm{ml}\right.$ overnight at $4^{\circ} \mathrm{C}$; Invitrogen, Eugene, OR).

Fluorescence images were acquired with a Bio-Rad confocal microscope (Bio-Rad, Hercules, CA). Excitation was performed at 488 and 647 $\mathrm{nm}$ wavelengths by a $\mathrm{Kr} / \mathrm{Ar}$ mixed-gas laser. We used standard T1/E2 filters with two detection filters of 522/35 nm (green pseudocolor) and $680 / 32 \mathrm{~nm}$ (red pseudocolor). These two wavelengths corresponded to the fluorescent dyes used in our experiments, Alexa 488 for revealing Neurobiotin (green) and Cy5 for 5-HT (red).

Intracellular recordings. Neuronal cell bodies in the isolated CNS were impaled with glass micropipettes (5-15 M $\Omega$ ) filled with $3 \mathrm{M} \mathrm{KCl}$. Membrane potential was measured using an Axoclamp 2B amplifier (Molecular Devices, Union City, CA), digitized with an ITC-16 analog-todigital/digital-to-analog computer interface (Instrutech, Great Neck, $\mathrm{NY}$ ), and recorded on a computer file with customized software written with Igor Pro 4.03 (WaveMetrics, Lake Oswego, OR). When examining synaptic transmission, the $\mathrm{MN}$ was hyperpolarized to $-70 \mathrm{mV}$ to prevent spiking. We took three tests at $15 \mathrm{~min}$ intervals to assess the baseline amplitude of the evoked EPSP in the MN. For each test, a single action potential was elicited in the SN (or L29 interneuron), and the resulting EPSP was measured in the MN. Synapses showing evidence of depression (i.e., $>25 \%$ decrease from the mean of the three tests) were discarded (10-20\% of the synapses). Short-term synaptic facilitation, as measured 5 min after tail-nerve stimulation, was expressed as the percentage increase from the baseline level (average of three pretests). When examining the effects of ipsilateral tail-nerve shock on SN-MN synaptic transmission, the recorded tail SN fired occasionally. Those cases were discarded to parallel our behavioral experiments, in which training and testing were performed on two different sites on the tail (i.e., the tail SNs activated by testing would not be expected to fire during training). Nerve shocks were delivered as one $2 \mathrm{~s}$ train of $15-20 \mathrm{~V}$ pulses at $40 \mathrm{~Hz}$. Such parameters have already been used for tail-nerve stimulation and provide a cellular analog of tail shock (Marinesco and Carew, 2002a).

Timing of experiments and seasonal effects. Several biological markers undergo a marked seasonal rhythm in Aplysia (Redman and Berry, 1991; Wayne, 2001). We should note that we repeatedly observed that 5-HT signals are greater when recorded during the fall than during the spring or summer, whereas other parameters such as duration of 5-HT release, time to peak, and threshold for nerve stimulation did not appear to vary (our unpublished observations). Differences in absolute 5-HT concentrations observed in experiments performed at different times of the year likely result from a seasonal variation (see, for example, Fig. 9). In contrast, the electrophysiological properties of the serotonergic neurons that we recorded did not appear to undergo a seasonal variation (i.e., there was no correlation between basal firing rate or the effects of nerve shocks and the time of the year). For this reason, electrophysiological recordings of serotonergic neurons were performed throughout the year with the exception of August and September, which is the mating season, whereas 5-HT release was mostly studied between October and February. There was sufficient overlap between the two sets of experiments to allow a comparison between 5-HT release data and the firing rate of serotonergic neurons.

Statistics. Data were presented as mean \pm SEM. Comparisons between two data groups were performed using the Student's $t$ test for equal or unequal variances, as determined by the $F$ test (significance level was $p<$ 0.05 ). For comparisons between three or more data groups, we used an ANOVA followed by a least significant difference (LSD) post hoc test $(p<0.05)$. Statistics software was the analysis tool pack of Microsoft Excel 2000 (Microsoft, Redmond, WA) and SPSS for Windows version 10 (SPSS, Chicago, IL).

The basal firing rate of serotonergic neurons was highly variable between cells and was standardized using the $Z$-score method (i.e., the variations in firing rate were expressed as the number of SDs above or below the mean for each cell that we recorded). Statistical analysis was performed by an ANOVA followed by an LSD post hoc test.

\section{Results}

\section{Effects of tail shock and head shock on the T-SW reflex}

Several previous studies have suggested that sensitization in Aplysia is not uniformly induced by stimulation anywhere on the body surface. Rather, stimulation of sites close to the training site is typically the most likely to induce sensitization (Scholz and Byrne, 1987; Erickson and Walters, 1988; Illich et al., 1994; Wainwright et al., 2002). To explore this feature of behavioral plasticity and, eventually, to understand how 5-HT contributes to it, we examined the modulation of the T-SW reflex by electrical stimulation of three sites on the body: (1) the ipsilateral side of the tail, which is close to the site used for testing the T-SW reflex, (2) the contralateral side of the tail, and (3) the head. We first compared the effects of tail shock to those of head shock. Electrical shocks were applied as shown in Figure $1 A$. Confirming previous results (Sutton et al., 2002), four tail shocks spaced by 15 min produced the following: (1) robust short-term memory for sensitization $(+74 \pm 21 \% ; n=16 ; p<0.01$, measured 5 min after the first shock), (2) intermediate-term memory $(+67 \pm 13 \%$; $p<0.01$, average measurements 15-45 min after the last shock), and (3) long-term memory $(+71 \pm 10 \% ; p<0.01,24 \mathrm{~h}$ after training).

Although head shock induced defensive responses such as inking, retraction, and locomotion, which were qualitatively similar to those evoked by tail shock, its effects on the T-SW reflex were very different. Five repeated head shocks produced robust inhibition of the T-SW reflex. Five minutes after the first shock, the T-SW reflex was inhibited $(-25 \pm 4 \% ; n=11 ; p<0.01)$ (Fig. 
$1 B)$. After the fifth shock, behavioral inhibition was even greater $(-29 \pm 5 \% ; n=11 ; p<0.01)$. Inhibition was reflected by significantly shorter T-SW reflex responses compared both to baseline and to controls that received no shock (Fig. $1 B$ ). This inhibition was no longer observed $1 \mathrm{~d}$ after training (Fig. $1 B$ ).

We also compared the effects of ipsilateral and contralateral tail shocks by applying electrical stimulation to one side of the tail (Fig. 1C). Interestingly, a single contralateral or ipsilateral tail shock produced similar short-term sensitization, as measured 5 min after the first shock (ipsilateral: $+29 \pm 10 \%, n=15, p=$ 0.02 ; contralateral, $+28 \pm 8 \%, n=10, p=0.01)$. In the intermediate time range (15-45 min after training), four spaced ipsilateral tail shocks produced significant sensitization $(+56 \pm 8 \%$; $n=15 ; p<0.01)$. After four contralateral shocks, however, no significant enhancement in the duration of the T-SW reflex was exhibited $(+11 \pm 7 \% ; n=10 ; p=0.1)($ Fig. $1 D)$. Consistent with the observations of Wainwright et al. (2002), ipsilateral tail shock induced significant long-term memory measured 24 and $48 \mathrm{~h}$ after training $(+40 \pm 10 \%, p<0.01$ and $+31 \pm 12 \%, p<0.01$, respectively; $n=15)$, but contralateral tail shock did not $(+19 \pm$ $11 \%, p=0.06$ and $-5 \pm 6 \%, p=0.92$, respectively; $n=10$ ).

These data confirm the view that sensitization is best induced in areas of the body close to the site of training: ipsilateral tail shock produced maximal short-term, intermediate-term, and long-term sensitization, whereas contralateral tail shock produced only short-term sensitization. In contrast, head shock produced inhibition of the T-SW reflex. Thus, these results also show that the T-SW reflex can be bidirectionally modulated by noxious stimuli, depending on the site of training.

\section{Modulation of identified synaptic sites}

To explore the cellular mechanisms underlying the differences we observed in behavioral modulation induced by noxious stimulation of different sites of the body, we examined the effects of cellular analogs of ipsilateral tail shock, contralateral tail shock, and head shock at two identified synaptic sites: (1) the tail SN-MN connection and (2) the L29-siphon MN connection (Fig. 2). We applied electrical stimulation to the ipsilateral or contralateral tail nerve (P9) as a cellular analog of ipsilateral or contralateral tail shock, and we stimulated a head nerve (anterior tentacular nerve) to mimic head shock (Fig. $2 \mathrm{~A}$ ). The neuronal circuit underlying the T-SW reflex involves (1) the tail SNs, (2) the siphon LFS motor neurons, and (3) a number of interneurons (INs) located in the pleural and abdominal ganglia. Several of these interneurons have been identified (Fig. 2B) (Cleary et al., 1995; Frost and Kandel, 1995; Barbas et al., 2003), but the interneuronal circuit is not yet fully characterized. In our cellular studies, we first examined the well identified tail SN-MN synapses (Fig. 2 B, site 1) (Cleary et al., 1998; Purcell et al., 2003). We also examined an important source of excitation to the siphon MNs, the abdominal L29 interneurons (Fig. 2 B, site 2). Each of these four to five neurons can convey up to $40 \%$ of the tail-evoked excitation to the LFS neurons (Hawkins and Schacher, 1989; Fischer and Carew, 1993). Thus, to begin to explore synaptic contributions to the behavioral plasticity that we observe (Fig. 1), we studied the effects of ipsilateral and contralateral tail-nerve shock and head-nerve shock on both tail SN-MN and L29-LFS synapses (Fig. $2 B$ ).

Activation of the tail SN-MN synapses (Fig. $2 B$, site 1 ) was very different after ipsilateral tail-nerve, contralateral tail-nerve, or head-nerve shock (Fig. $3 A-C$ ). None of the three shocks produced action potentials in tail SNs (see Materials and Methods). Tail MNs, however, responded very differently to the different
A. Anterior tentacular nerve

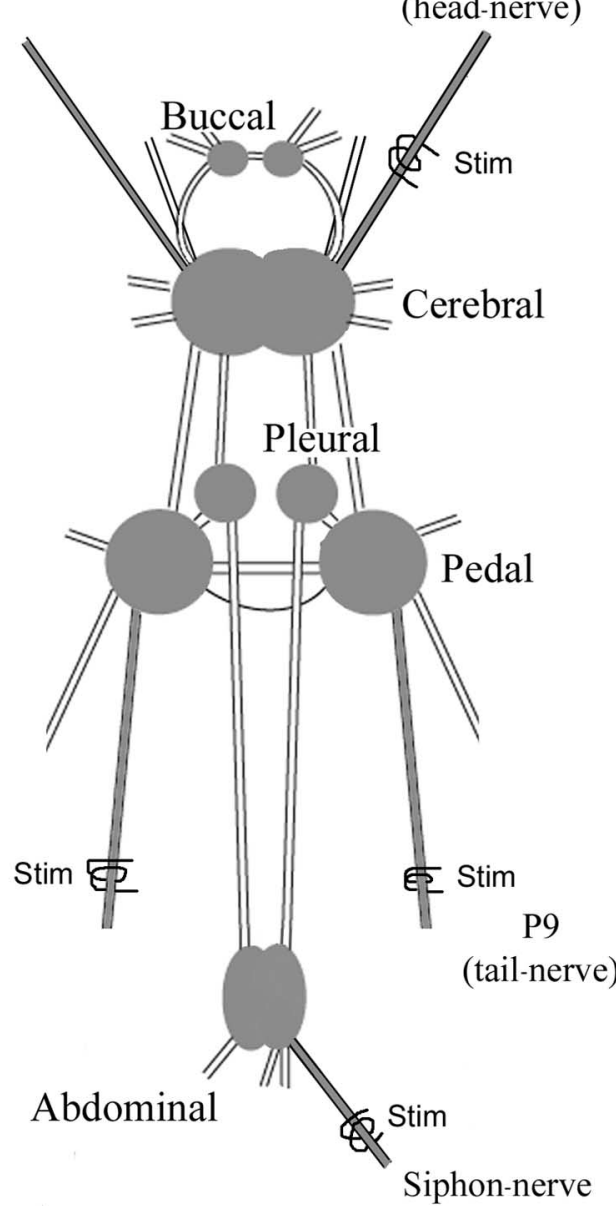

B.

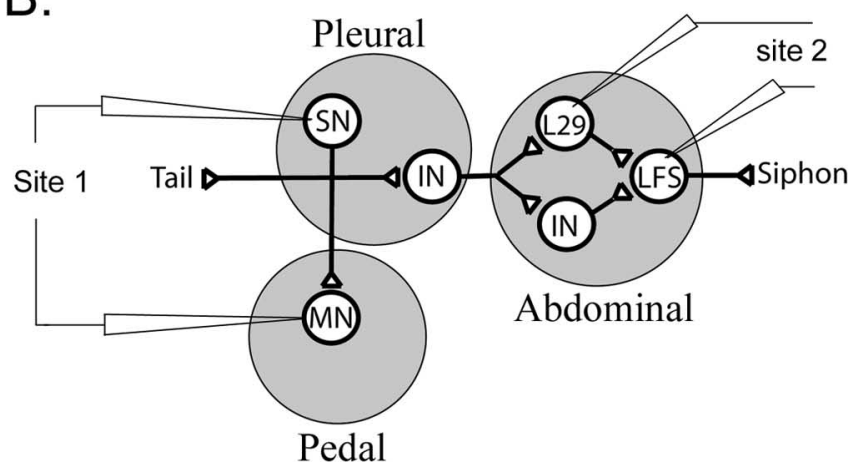

Figure 2. Cellular analysis of the modulation of the T-SW reflex.A, Schematic representation of the Aplysia CNS. We used an electrical stimulation (Stim) of the anterior tentacular nerve (head nerve) to mimic the effects of head shock and stimulated the ipsilateral or contralateral P9 (tail nerve) as a cellular analog of ipsilateral or contralateral tail shock. B, Schematic representation of the putative neuronal circuit underlying the T-SW reflex. A tactile stimulation of the tail activates tail SNs that contact tail MNs and INs located in the pleural ganglion. These interneurons contact other interneurons in the abdominal ganglion, some of which have been identified as the L29 subtype. Both L29s and other abdominal interneurons transmit excitation to LFS motor neurons that contribute to siphon retraction (Cleary et al., 1995; Frost and Kandel, 1995; Barbas et al., 2003).

stimuli. They fired a burst of action potentials in response to ipsilateral tail-nerve shock, whereas they were hyperpolarized by contralateral tail-nerve shock (Fig. $3 A, B$ ). Head shock usually produced a slight subthreshold depolarization (Fig. 3C) or no 
effect. In examining synaptic plasticity, ipsilateral tail-nerve stimulation produced maximal facilitation of tail SN-MN synapses 5 min after the shock $(+147 \pm 19 \%$; $n=7 ; p<0.01)$. Contralateral tail-nerve shock produced less robust facilitation $(+52 \pm 12 \% ; n=6 ; p<0.01)$ that was significantly reduced compared with ipsilateral tail-nerve shock $(p<0.01)$ (Fig. $3 D)$. Finally, head-nerve shock did not induce any significant change in EPSP size $(+21 \pm 19 \% ; n=6 ; p=0.1)$ (Fig. 3D).

We also determined the effects of comparable tail-nerve and head-nerve shock on L29-LFS synapses (Fig. 2B, site 2). There are two subtypes of L29 interneurons: the $\mathrm{L} 29_{\mathrm{A}}$ type is more responsive to head stimuli, whereas the L29 $\mathrm{B}$ type fires preferentially in response to tail/siphon stimuli (Hawkins and Schacher, 1989). Because they contribute more to the T-SW reflex, we specifically focused on the $\mathrm{L} 29_{\mathrm{B}} \mathrm{S}$, which can be found laterally on the right abdominal hemiganglion (Hawkins and Schacher, 1989; Frost and Kandel, 1995). The L29 interneurons that we selected fired more intensely in response to tailnerve shock than to head-nerve shock, confirming that they belonged to the L29 subtype (Fig. $4 A, B$ ). The siphon LFS MNs also tended to fire somewhat more action potentials in response to tail-nerve shock than head-nerve shock (Fig. 4A,B), in agreement with the behavior of intact animals, in which tail shock typically produces a flaring siphon response involving LFSs, whereas head shock produces a constricting response that is thought to rely on other subtypes of siphon MNs (LBS and RDS subtypes) (Hickie and Walters, 1995). In agreement with previous studies (Fang and Clark, 1996), L29-LFS synapses were facilitated in response to tail-nerve shock $(+43 \pm 8 \% ; n=6 ; p<0.01)$. There was no difference between electrical shocks applied to the left or the right tail nerve (right tail nerve, $+44 \pm 15 \%$; left tail nerve, $+43 \pm 12 \% ; n=3 ; p=0.92$ ), confirming previous data (Hawkins and Schacher, 1989; Fang and Clark, 1996). In contrast, L29-LFS synapses were inhibited in response to head-nerve shock $(-38 \pm$ $8 \% ; n=7 ; p<0.01$ ) (Fig. $4 B, C$ ).

Although there certainly are additional neuronal elements that could also contribute to the modulation of the T-SW reflex by head or tail shock, synaptic plasticity at tail SN-MN and L29-LFS synapses corresponded well with the behavioral data observed in intact animals. First, head-nerve shock inhibited L29-LFS synapses and did not significantly change tail SN-MN connections, which is in good agreement with
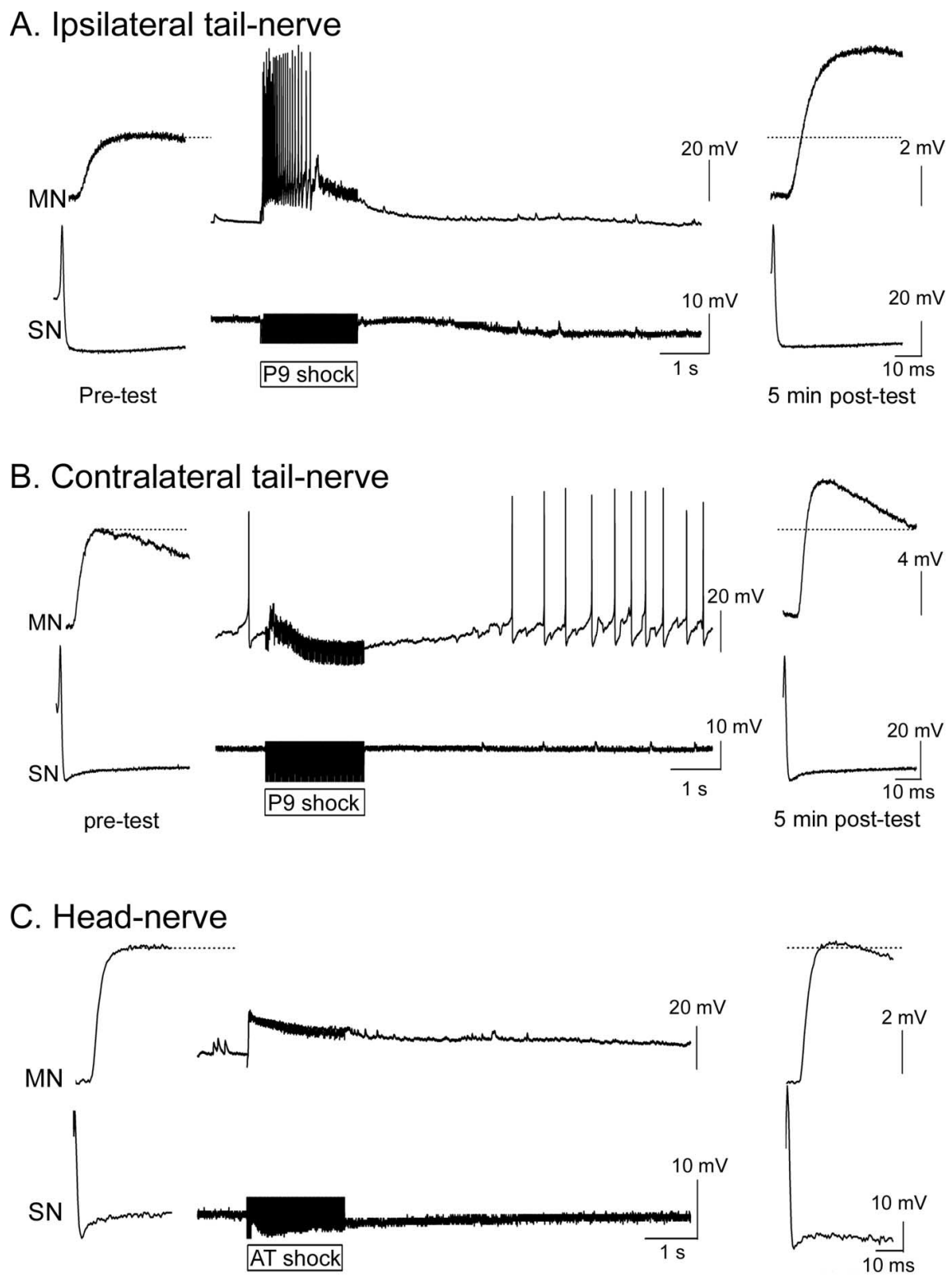

Pre-test

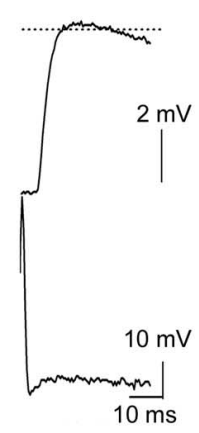

5 min post-test

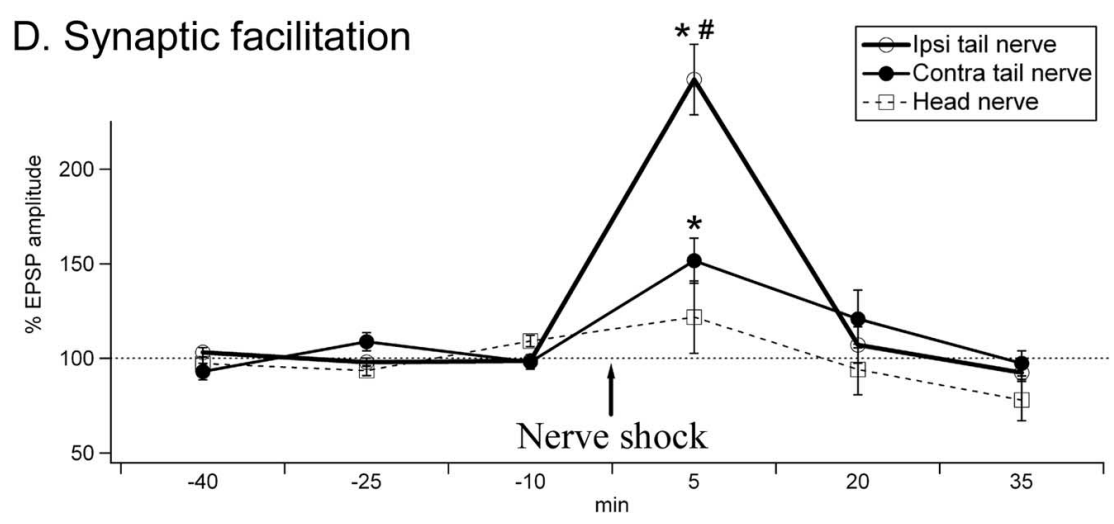

Figure 3. Tail SN-MN synaptic plasticity in response to ipsilateral tail-nerve, contralateral tail-nerve, or head-nerve shock. $A-C$, Sample traces of tail SN-MN synapses before, during, and 5 min after nerve shock. AT, Anterior tentacular nerve. $\boldsymbol{D}$, Effects of ipsilateral (Ipsi) tail-nerve, contralateral (Contra) tail-nerve, and head-nerve shock. Synaptic facilitation was maximal after ipsilateral tail-nerve shock. Significant facilitation was also present contralateral to tail-nerve stimulation. However, no significant facilitation was found after head shock (Ipsi tail nerve, $n=7$; Contra tail nerve, $n=6$; head nerve, $n=7$ ). *Significantly different from baseline. "Significant difference between Ipsi tail-nerve and Contra tail-nerve (and head-nerve) shock. Error bars represent SEM. 


\section{A. Tail-nerve shock}

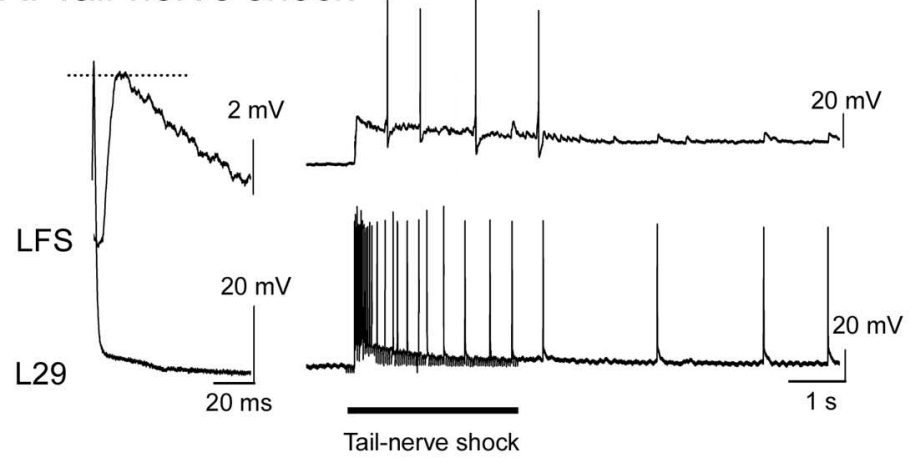

B. Head-nerve shock

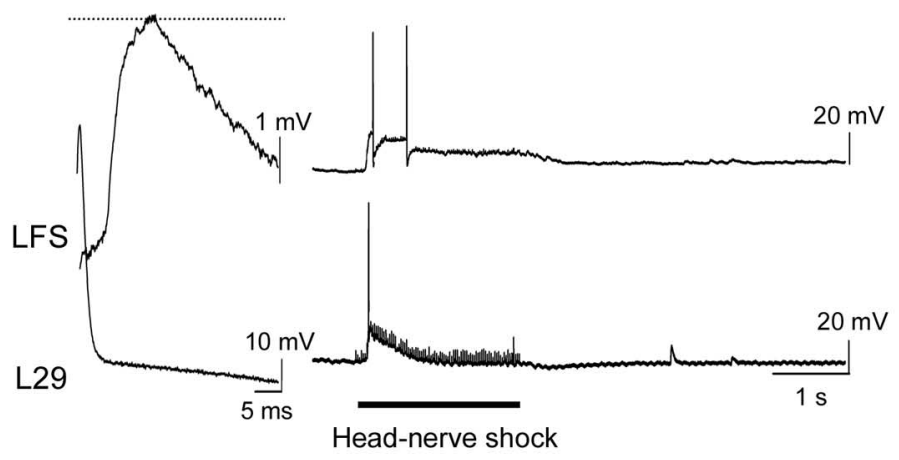

\section{Synaptic facilitation}

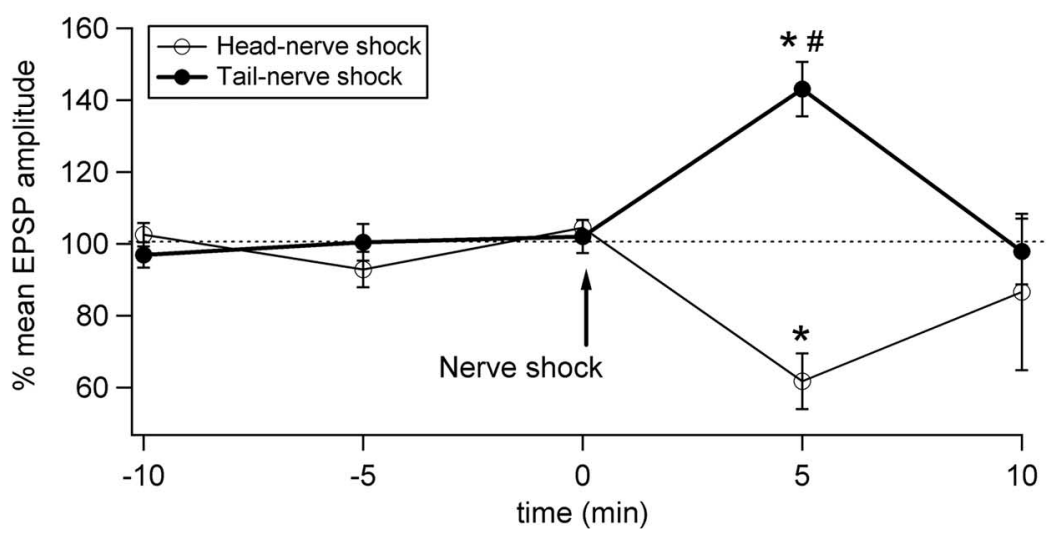

Figure 4. L29-LFS synaptic plasticity in response to head-nerve and tail-nerve shocks. $\boldsymbol{A}, \boldsymbol{B}$, Sample traces of L29-LFS synapses before, during, and 5 min after tail-nerve $(\boldsymbol{A})$ or head-nerve shock $(\boldsymbol{B})$. L29 neurons fired a train of action potentials only in response to tail-nerve shock. $C$, Mean EPSP amplitude in response to head-nerve or tail-nerve shock. Tail-nerve shock facilitated L29 -LFS synapses, whereas head-nerve shock inhibited them. *Significantly different from baseline. "Significant difference between tail-nerve and head-nerve shock. Error bars represent SEM.

the observed behavioral inhibition. Second, contralateral tailnerve shock facilitated L29-LFS synapses and induced modest facilitation of tail SN-MN connections, in accordance with modest, transient sensitization in behavioral experiments. Third, ipsilateral tail-nerve shock facilitated L29-LFS synapses and produced robust facilitation at tail SN-MN connections, consistent with maximal sensitization in intact animals.

\section{Effects of tail and head-nerve shocks on the firing rate of serotonergic neurons}

Serotonergic neurons have been implicated in both sensitization (Brunelli et al., 1976; Glanzman et al., 1989; Mackey et al., 1989;
Marinesco and Carew, 2002a) and behavioral or synaptic inhibition (Fitzgerald and Carew, 1991; Bristol et al., 2001; Marinesco et al., 2004b). To understand the contribution of the serotonergic system to the modulation of the T-SW reflex by ipsilateral tail shock, contralateral tail shock, or head shock, we recorded the activity of these cells after they were prelabeled with 5,7-DHT. In a previous study, we showed that after $\sim 2$ months, 5,7-DHT administration produces a dark brown pigmentation that colocalizes with 5-HT immunoreactivity (Marinesco et al., 2004a). Using this method, we could identify and record from serotonergic neurons within the pedal and abdominal ganglia. An example of such recordings is shown in Figure $5 B$. The neurons from which we recorded were always injected with Neurobiotin at the end of the recording and processed for 5-HT immunohistochemistry (see Materials and Methods) to confirm their serotonergic phenotype (Fig. 5A1-A3). We continuously recorded from a total of 10 cells (seven in the pedal ganglion and three in the right $\mathrm{B}$ cluster of the abdominal ganglion) from at least $10 \mathrm{~min}$ before until 25 min after tail- or head-nerve shock. The firing rate (in spikes per $1 \mathrm{~min}$ bin) of the neuron was then computed and standardized ( $Z$-score; see Materials and Methods) to its baseline level before shock (Fig. 6).

We should note that many of the neurons that we recorded [such as the parapodia-opener phase-like (POP-like) neuron in Fig. 5] project to the periphery. However, they may still be able to modulate the T-SW reflex either by releasing 5-HT within the CNS by en-passant boutons or by modulating the terminals or neuromuscular junctions of sensory neurons at the periphery (Billy and Walters, 1989; Lotshaw and Lloyd, 1990; Laurienti and Blankenship, 1997).

Both tail- and head-nerve shock significantly increased the firing rate of all of the serotonergic neurons from which we recorded (tail-nerve shock: $Z$-score = $19.7 \pm 4.8, p<0.01$; head-nerve shock: $Z$-score $=6.1 \pm 1.2 ; p<0.01)($ Fig. $6 A)$. Because there was no difference between the response of pedal and abdominal serotonergic neurons to head- or tail-nerve shock, we pooled the data obtained from these two ganglia. The increase in firing rate lasted $\sim 8 \mathrm{~min}$ in both cases. However, tail-nerve shock produced a significantly larger increase in firing rate than head-nerve shock ( $p<0.05$ during 2 min after shock) (Fig. 6A). In a previous study (Marinesco et al., 2004a), we similarly recorded the response of prelabeled serotonergic neurons in the same areas. Among the cells from which we recorded, 26 were tested for input from both ipsilateral and contralateral tail-nerve shock. Their responses to ipsilateral or contralateral tail-nerve shock were not analyzed in the previous study. When standard- 

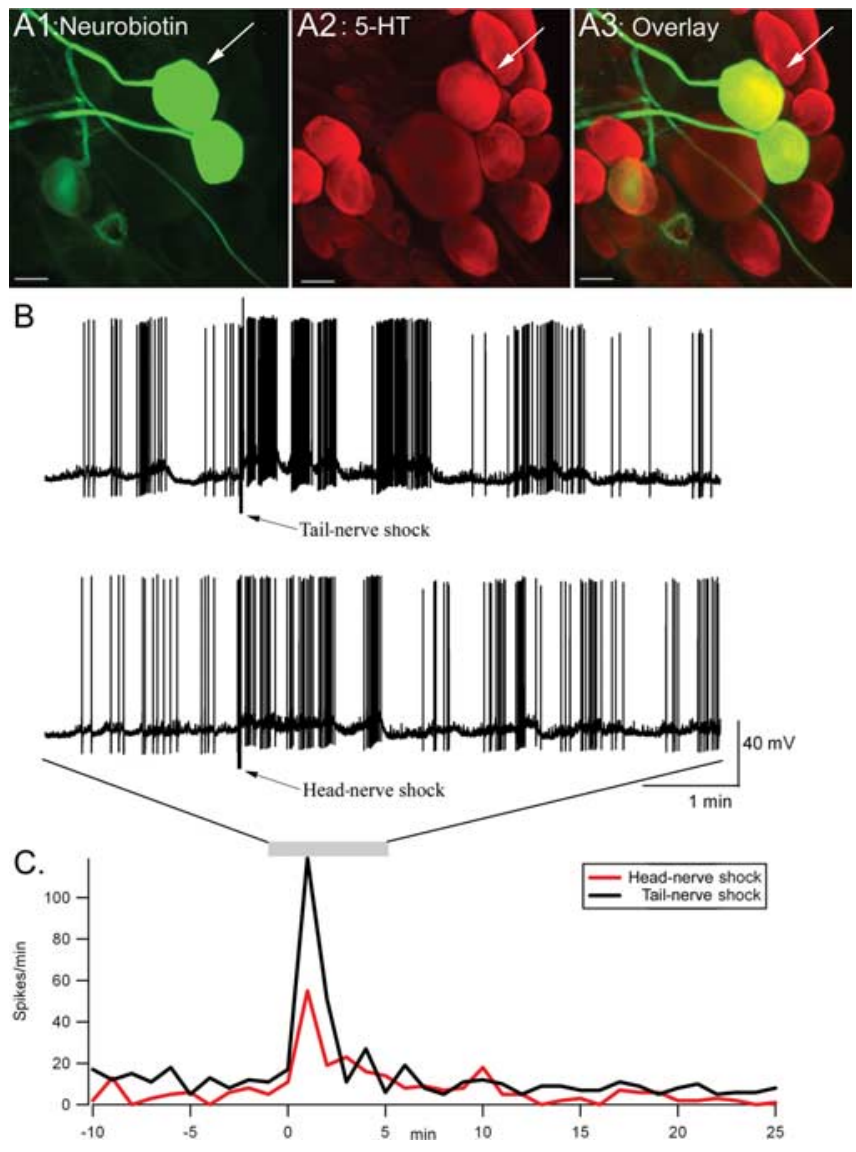

Figure 5. Effects of head-nerve versus tail-nerve shock on the firing rate of an identified serotonergic neuron. A1-A3, Immunohistochemical characterization of two serotonergic neurons in the pedal ganglion (A1, Neurobiotin staining; $\boldsymbol{A} \mathbf{2}, 5$-HT immunoreactivity; $\boldsymbol{A} \mathbf{3}$, overlay). Scale bars, $100 \mu \mathrm{m}$. $\boldsymbol{B}$, Intracellular recording of one serotonergic neuron (arrow in $\boldsymbol{A}$ ). Recording is shown between $2 \mathrm{~min}$ before and $5 \mathrm{~min}$ after tail-nerve (top trace) or head-nerve shock (bottom trace). The bursting pattern of this neuron, as well as its localization within the pedal ganglion, is typical of a POP-like neuron. C, Firing rate (in spikes per $1 \mathrm{~min}$ bin) during the entire duration of the recording. The increase in firing rate is more pronounced after tail-nerve shock than after head-nerve shock.

izing and comparing the effects of both tail-nerve shocks in these 26 neurons, we found a very similar increase in firing rate: ipsilateral $Z$-score $=13.2 \pm 2.1, p<0.01$; contralateral $Z$-score $=$ $15.9 \pm 2.7, p<0.01$ (Fig. $6 B$ ).

These results indicate that the firing rate of serotonergic neurons is differentially regulated by head-nerve shock or tail-nerve shock. However, these differences cannot fully account for the modulation of the T-SW reflex observed in intact animals, because ipsilateral and contralateral tail shocks induced sensitization with very different amplitudes and time courses (Fig. 1C,D), despite quite similar effects of both ipsilateral and contralateral nerve shock on the electrical activity of serotonergic neurons (Fig. $6 B)$.

\section{Nerve shocks evoke distinct patterns of 5-HT release in the CNS}

Considering the dissociation between 5-HT neurons firing rates, which seem rather uniformly increased by ipsilateral or contralateral tail-nerve shock, and the differential behavioral effects that we observed, we reasoned that it may be possible that 5-HT modulation of the T-SW reflex relies on specific patterns of 5-HT release that could not be directly estimated from the firing rate of the serotonergic cell bodies from which we recorded. To explore
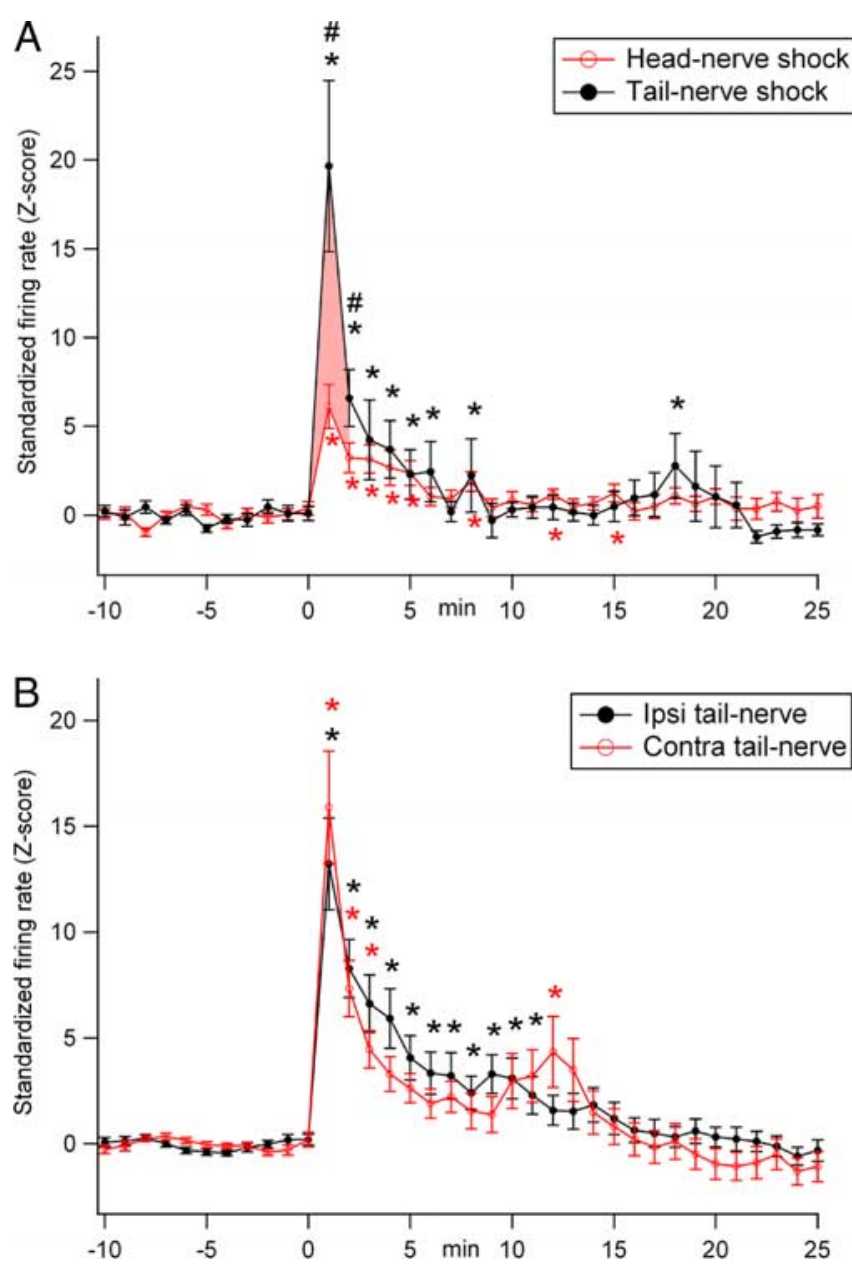

Figure 6. Average effects of electrical stimulation of head nerve, ipsilateral tail nerve, or contralateral tail nerve on the firing rate of serotonergic neurons in the pedal and abdominal ganglia. $\boldsymbol{A}$, Comparison between head-nerve shock and tail-nerve shock in 10 serotonergic neurons. Tail-nerve and head-nerve shock both significantly elevated the firing rate of all of the recorded serotonergic neurons. This elevation was significantly more pronounced after tailnerve shock than after head-nerve shock during the first 2 min after shock (pink shaded area). $\boldsymbol{B}$, Comparison between ipsilateral (Ipsi) and contralateral (Contra) tail-nerve shock in 26 serotonergic neurons. Both nerve shocks significantly elevated the firing rate of serotonergic neurons, without any significant difference between ipsilateral and contralateral tail-nerve shock. ${ }^{*}$ Sig-

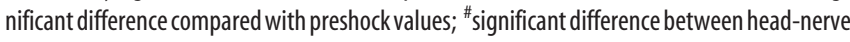
and tail-nerve shock $(p<0.05)$. Error bars represent SEM.

this possibility, we used carbon fiber microelectrodes, which we have shown can readily detect 5-HT release within Aplysia ganglia (Marinesco and Carew, 2002a,b). To better understand the serotonergic contribution to the modulation of the T-SW reflex, we measured the variations of the 5-HT extracellular concentration in response to ipsilateral and contralateral tail-nerve shock and head-nerve shock (1) in the pleural ganglion, in the vicinity of tail $\mathrm{SN}$ cell bodies, (2) in the pedal ganglion, close to tail SN-MN synapses, and (3) in the abdominal ganglion, near the siphon $\mathrm{MN}$ cell bodies.

5 -HT release was detected by implanting our electrodes underneath the tail SNs in the pleural ganglion (see Fig. 9A1) or underneath the tail MNs in the pedal ganglion. The 5-HT release profile in the pleural and pedal ganglia was very similar and reached similar concentrations (Fig. $7 B, C$ ) for each nerve that we stimulated (ipsilateral or contralateral tail nerve or head nerve). 5-HT release was maximal after ipsilateral tail-nerve shock and reached a peak concentration after $4-6 \mathrm{~s}$ of $\sim 88 \pm 18 \mathrm{nM}$ in the 
A.

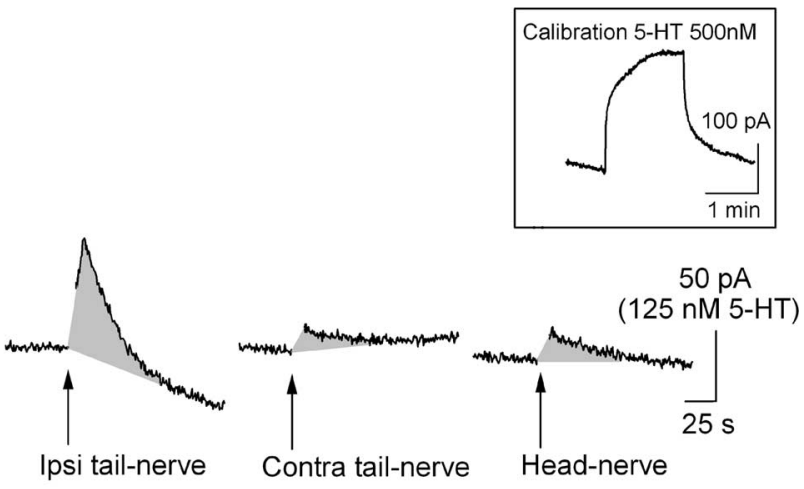

B. Pleural

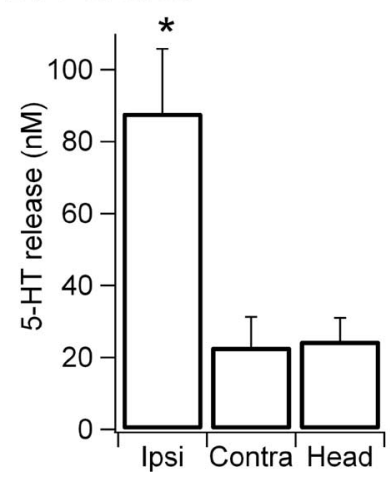

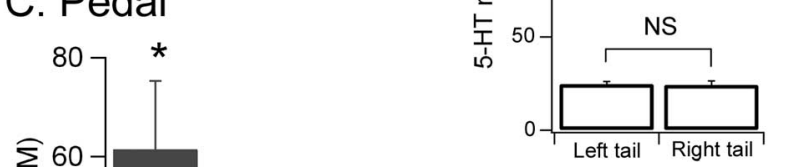
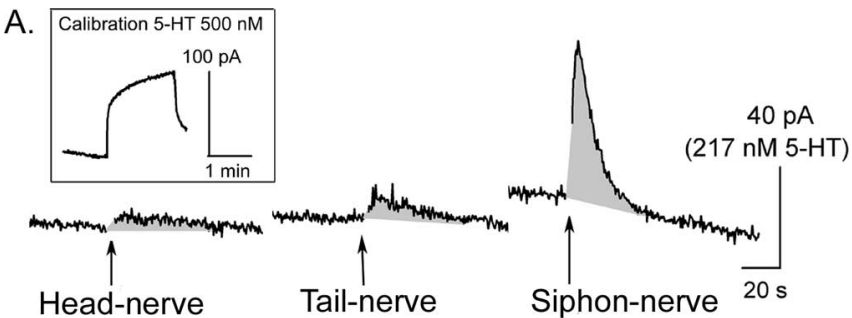

B.

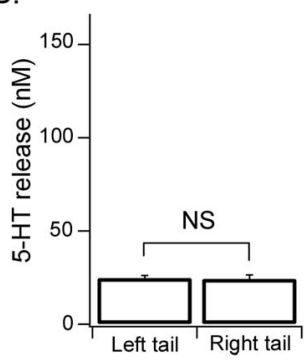

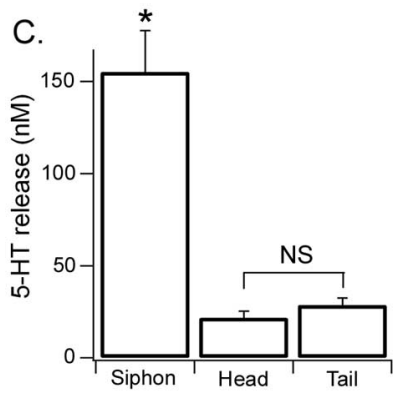

Figure 8. 5 -HT release in the abdominal ganglion. $\boldsymbol{A}$, Example of chronoamperometric traces showing 5-HT release (gray shaded area) after head-nerve, tail-nerve, and siphon-nerve shock. $\boldsymbol{B}, 5$-HT release was equally low after left or right tail-nerve shock. $\boldsymbol{C}, 5$-HT release was maximal after siphon-nerve shock but significantly lower after tail-nerve or headnerve shock $(n=7)$. * Significant difference compared with head- and tail-nerve shock. Error bars represent SEM.

lease in the vicinity of siphon SNs or MNs (LEs and LFSs). Indeed, siphon-nerve shock produced significantly more 5-HT release than the other two stimuli $(p<0.01)$.

These data show that, although ipsilateral and contralateral tail-nerve shock, as well as head-nerve shock, increase the firing rate of a large number of serotonergic neurons, the spatial pattern of 5-HT release is different among the three stimuli that we studied. Most strikingly, ipsilateral and contralateral tail-nerve shock produced similar effects on the firing rate of serotonergic neurons but induced different patterns of release in the CNS. In addition, the duration of 5-HT release (30-40 s) was much shorter than the duration of increased firing rate in our sample of serotonergic neurons. Therefore, the firing rate of a large population of serotonergic neurons is not sufficient to accurately predict the pattern of 5-HT release in the CNS.

\section{Local modulatory fields of 5-HT release}

Taking advantage of the high spatial resolution of our carbonfiber microelectrodes, we next investigated the spatial organization of 5-HT release in the pleural and abdominal ganglia. We first implanted our microelectrodes near the tail SN cell bodies, in the pleural ganglion (Fig. 9A1, field 1). We then removed the tip of the electrode from the ganglion and implanted it again $\sim 500$ $\mu \mathrm{m}$ more rostral, near the area in which the pleural-abdominal connective originates (Fig. 9A1, field 2). Tail-nerve shock produced maximal 5-HT release in field 1 ( $48 \pm 8 \mathrm{nM})$ (Fig. 9A2,A3) and significantly less in field $2(19 \pm 4 \mathrm{nM} ; p<0.01)$ (Fig. $9 A 2, A 3)$. This decrease was not attributable to run down of the microelectrode or the preparation, because when we repositioned the microelectrode in field 1 again, we recorded 5-HT signals that were not significantly different from the first recording (59 $\pm 19 \mathrm{nM} ; n=6$ ) (Fig. 9A2,A3). These data are similar to those we obtained in the pedal ganglion in a preceding study (Marinesco and Carew, 2002a), which showed that 5-HT release was maximal in the vicinity of tail MNs and undetectable $500 \mu \mathrm{m}$ more caudal. 
A1

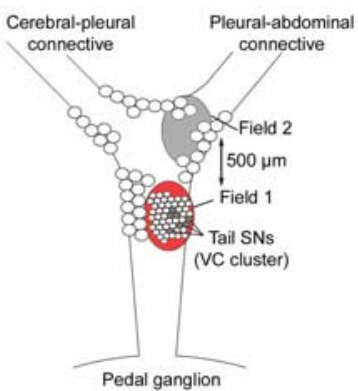

B1
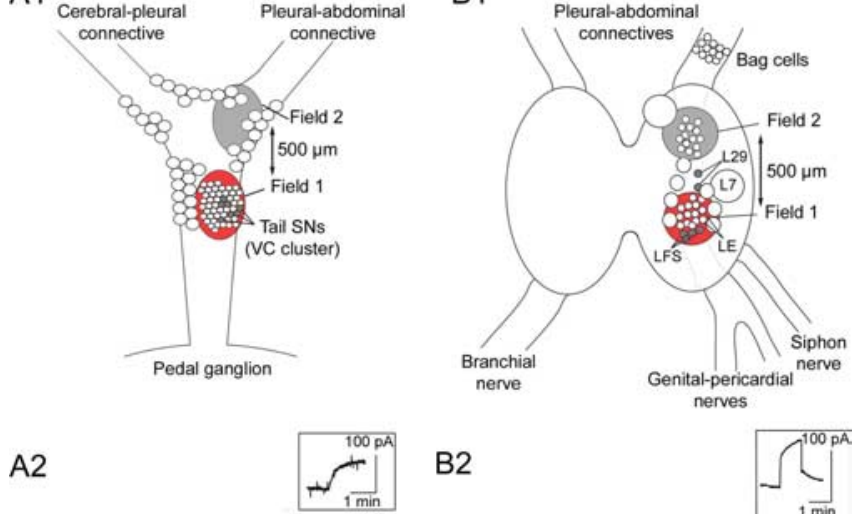

B2
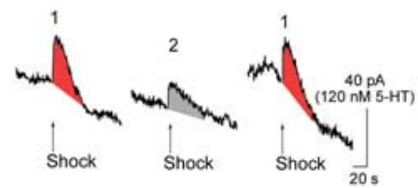

A3

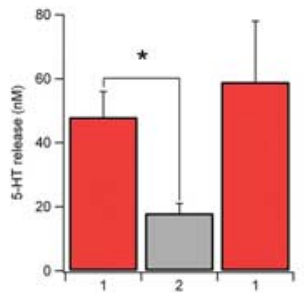

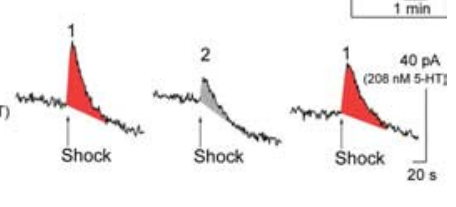

B3

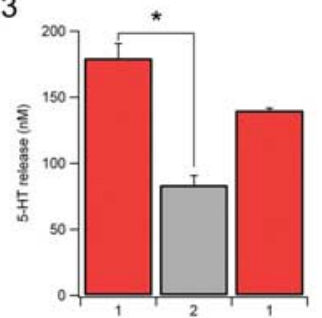

Figure 9. Functional organization of 5-HT release. Location of the modulatory fields (in red) in which 5-HT release was detected (A1, pleural ganglion; $\boldsymbol{B} 1$, abdominal ganglion). $\boldsymbol{A 2}, \mathbf{B 2}$, Sample traces of 5 -HT signals recorded in the pleural and abdominal ganglia, respectively. Insets, Calibration experiments performed in a 5-HT standard solution at $200 \mathrm{~nm}$ (A2) or $500 \mathrm{~nm}$ (B2). A3, B3, Mean 5-HT release detected in the two different modulatory fields within the pleural and abdominal ganglia. 5-HT release was maximal in the vicinity of tail SNs in the pleural ganglion and close to the LFS siphon MNs in the abdominal ganglion ( $n=6$ pleural and abdominal). VC, Ventrocaudal. *Significant difference between fields 1 and 2 . Error bars represent SEM.

We performed a similar experiment in the abdominal ganglion, by implanting our electrodes near siphon MNs, in the caudal portion of the right hemiganglion (Fig. 9B1, field 1) and $\sim 1$ $\mathrm{mm}$ more rostral (Fig. 9B1, field 2). Siphon-nerve shock produced maximal 5-HT release in field $1(179 \pm 11 \mathrm{~nm})$ (Fig. $9 B 2, B 3)$ but evoked significantly less 5 -HT release in field 2 ( $83 \pm$ $7 \mathrm{~nm} ; p<0.01$ ) (Fig. 9B2,B3). As above, larger 5-HT signals were recorded when we implanted the electrode again in field 1 (140 \pm $2 \mathrm{~nm} ; n=6 ; p=0.07$, ANOVA; $p=0.04$, Student's $t$ test) (Fig. $9 B 2, B 3)$. The difference in absolute 5 -HT concentrations estimated in the pleural and abdominal ganglia is probably attributable to seasonal differences in 5-HT release (see Materials and Methods).

These data indicate that 5-HT release is not uniform within a single ganglion. Rather, steep concentration gradients can be generated between areas separated by as little as $500 \mu \mathrm{m}$ to $1 \mathrm{~mm}$. These gradients define hot spots of maximal 5-HT release, or modulatory fields (Marinesco and Carew, 2002a), that can account in principle for the fact that global firing of 5-HT neurons in response to noxious input can give rise to quite local modulatory events. Thus, depending on the site of the input, qualitatively similar activation of 5-HT neurons can give rise to different forms of behavioral and synaptic modulation.

\section{Discussion}

In this paper, we provide evidence for surprisingly restricted serotonergic hot spots or modulatory fields generated in response to neural analogs of the noxious stimuli that are typically used to induce memory for sensitization in Aplysia. These local gradients in extracellular 5-HT concentration suggest that, despite widespread firing of serotonergic neurons in response to noxious input, 5-HT release is regulated by local mechanisms and that the serotonergic system is capable of independently modulating different synaptic sites within a neural circuit. At the behavioral level, this finding can, in principle, explain why 5-HT can contribute to short-term (contralateral tail shock) or long-term sensitization (ipsilateral tail shock) or behavioral inhibition (head shock) after noxious stimuli applied to different sites on the body.

\section{Local patterns of 5-HT release}

The fact that 5-HT release is not uniform within the CNS has been suggested by experiments in several invertebrate models. For example, in the Aplysia and leech CNS, exogenous application of 5-HT is not always equivalent to stimulation of endogenous 5-HT release (Fox and Lloyd, 1998; Marinesco et al., 2004b; Calvino et al., 2005). These data supported the inference that exogenous 5-HT, even in physiological concentrations, can activate receptors that are not a natural target of 5-HT when it is endogenously released (for example, by nerve stimulation). This in turn suggests that 5-HT is not released in equal amounts throughout the CNS. In the present study, using the high spatial and temporal resolution of chronoamperometric techniques, we were able to quantify these disparities in 5-HT release. We found sharp differences in extracellular 5-HT concentrations within a few hundred micrometers in the neuropil of pleural and abdominal ganglia, extending our previous findings that revealed similar gradients in the pedal ganglion (Marinesco and Carew, 2002a).

In the two modulatory fields that we investigated, one in the pleural ganglion and the other in the abdominal ganglion, 5-HT release was regulated independently: in the pleural ganglion, tailnerve shock produced maximal 5-HT release near the tail SNs, but in the abdominal ganglion, the same input evoked only moderate release. Interestingly, we could record 5-HT signals of the same magnitude in both modulatory fields (after siphon-nerve shock in the abdominal ganglion and after ipsilateral tail-nerve shock in the pleural ganglion). This result shows that the differences in extracellular 5-HT concentrations between these two areas are not attributable to disparities in the density of 5-HT release sites but rather that both areas are innervated by serotonergic fibers with equal potential for 5-HT release.

What are the mechanisms responsible for these local hot spots of 5-HT release? Our recordings of prelabeled serotonergic neurons showed that they increased their firing rate in a rather uniform manner to the different nerve shocks that we tested. However, this aspect of serotonergic activation is not sufficient to account for the differences in 5-HT release that we measured in the pleural and pedal ganglia for three reasons: (1) ipsilateral and contralateral tail-nerve shock produce the same increase in firing rate but give rise to different 5-HT release profiles in the pleural and pedal ganglia, (2) the increase in firing rate significantly outlasts the increased 5-HT release detected in the ganglia ( 8 min vs $40 \mathrm{~s}$ ), and (3) although tail-nerve shock produced significantly more firing in 5-HT neurons throughout the CNS, extracellular 5-HT concentration was enhanced only in discrete modulatory fields centered around tail SNs and MNs. It is not possible to record exhaustively from all serotonergic neurons in the Aplysia CNS, and thus, we cannot exclude the possibility that specific 
5-HT neurons increase their firing for only $40 \mathrm{~s}$. Some of them could project to tail SNs in the pleural ganglion and respond only to tail-nerve shock, whereas others would innervate siphon MNs in the abdominal ganglion and respond only to siphon-nerve shock. Although such an anatomical organization could in principle produce modulatory fields similar to those revealed in the present study, it does not seem compatible with the notion that pleural and abdominal ganglia mostly share common 5-HT inputs that originate from $\mathrm{CC} 3 / \mathrm{CB} 1$ neurons in the cerebral ganglion (Mackey et al., 1989; Wright et al., 1995; Xin et al., 2001).

A more parsimonious explanation would be that 5-HT release is modulated by neurotransmitters acting locally on serotonergic fibers. In our system, presynaptic modulation of 5-HT release could be exerted by primary SN terminals. Indeed, in the two Aplysia ganglia that we investigated, 5-HT release was maximal in the vicinity of the terminals originating from primary SNs whose axons project in the stimulated nerve. In the modulatory field that we studied in the abdominal ganglion, siphon SNs (LEs) lie in close proximity to the siphon MNs (Fig. 9B1) (Frost and Kandel, 1995; Illich and Walters, 1997). In this area, 5-HT release was maximal after siphon-nerve shock, which activates LE siphon SNs. Similarly, after tail-nerve shock, areas surrounding tail SN terminals ipsilateral to the stimulation (pleural and pedal ganglia), which are activated by the shock, were the only serotonergic hot spots that we observed. Therefore, there is a strong correlation between primary SN activation and amplification of 5-HT release. In addition, using confocal microscopy, Zhang et al. (2003) showed contacts between tail SNs and serotonergic fibers. Primary SNs therefore appear to be in an ideal position to modulate or trigger 5-HT release locally through axo-axonic contacts. This organization would allow primary SNs that are strongly activated by noxious stimuli to directly trigger 5 -HT release onto themselves and their neighbors, thereby increasing their excitability, facilitating their synaptic contacts, and eventually, sensitizing their reflex pathways.

Interestingly, Zhang et al. (2003) also determined that serotonergic processes usually make contact with SNs or MNs at sites located at a distance from $\mathrm{SN}-\mathrm{MN}$ synapses. They proposed that serotonergic transmission in this area is essentially volumic or paracrine (i.e., 5-HT travels over distances of a few hundred micrometers before reaching its postsynaptic targets) [for review on paracrine transmission, see Bunin and Wightman (1999)]. This anatomical configuration would make serotonergic transmission extremely sensitive to reuptake mechanisms. Rapid 5-HT reuptake along the diffusion path could create a nonlinear relationship between the extracellular 5-HT concentration and the firing rate of serotonergic neurons (i.e., significant 5-HT spillover is only observed for high firing rates). Therefore, the increase in extracellular 5-HT concentration detected in response to nerve shock (lasting 30-40 s), could reflect the peak of the increase in the firing rate of serotonergic neurons (otherwise lasting a total of $8 \mathrm{~min}$ ). Alternatively, it is possible that 5-HT release quickly diminishes because of depletion of the releasable pool, despite continued firing of serotonergic neurons.

\section{Serotonergic regulation of behavioral and synaptic plasticity} In the defensive reflexes of Aplysia, 5-HT exerts both sensitizing effects (Brunelli et al., 1976; Castellucci and Kandel, 1976; Glanzman et al., 1989) and inhibitory effects (Fitzgerald and Carew, 1991). This complex regulation is probably mediated by multiple 5-HT receptors with diverse ionic and biochemical effects (Barbas et al., 2003). Our data indicate that three noxious stimuli that activate serotonergic neurons and evoke 5 -HT re-

\section{A. Head shock}
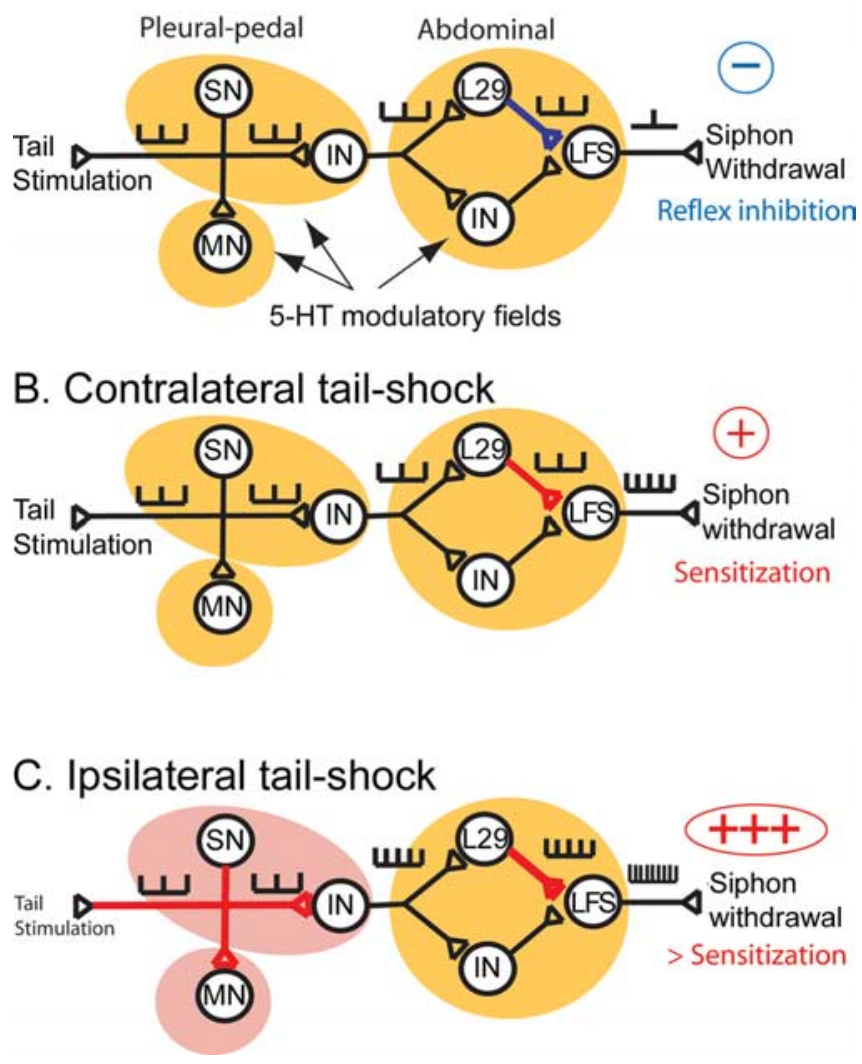

Figure 10. Simplified circuit of the tail-induced siphon-withdrawal reflex and its modulation by sensitizing or inhibitory stimuli. In the T-SW reflex, tactile information from the tail is transmitted to tail SNs and processed by several INs toward the LFS siphon MNs, which are responsible for siphon withdrawal. Tail SNs make excitatory synapses onto tail MNs and onto as yet unidentified pleural INs. These INs then contact L29 interneurons and other abdominal INs, which process the information in parallel. L29 INs make excitatory synapses onto LFS siphon MNs. 5-HT has different effects on synaptic efficacy within this circuit. In the pleural-pedal ganglia, it facilitates SN-MN synapses (and probably SN-IN synapses), whereas it inhibits L29 - LFS synapses in the abdominal ganglion. 5-HT is released in the pleural-pedal and abdominal ganglia at different concentrations depending on the body site at which noxious stimulation occurs. $A$, Head shock releases low amounts of 5-HT (indicated by yellow modulatory fields) in both the pleural-pedal ganglia and the abdominal ganglion, resulting in inhibition of L29LFS synapses and behavioral inhibition. $\boldsymbol{B}$, Contralateral tail shock also induces low 5-HT release (yellow modulatory fields) but activates L29 interneurons, resulting in PTP at L29-LFS synapses and short-lasting behavioral sensitization. $C$, Ipsilateral tail shock induces a large 5-HT release (indicated by red modulatory fields) in the pleural and pedal ganglia, resulting in the facilitation of synapses between SNs and the postsynaptic targets of 5-HT. Synaptic facilitation in the pleural and pedal ganglia and PTP at L29-LFS synapses produce longer-lasting behavioral sensitization. Thus, each of the three noxious stimuli that we tested result in different spatial patterns of 5-HT. This local regulation of 5-HT release within modulatory fields allows specific encoding of sensitization or inhibition in response to noxious stimuli.

lease in the CNS (ipsilateral tail shock, contralateral tail shock, and head shock) induce diverse effects on the T-SW reflex, ranging from sensitization to net behavioral inhibition. Serotonergic modulation appears to participate in the neural encoding of these input pathways, at least at the level of tail SNs and L29 interneurons. Because 5-HT facilitates SN-MN synapses (Brunelli et al., 1976; Mercer et al., 1991) and inhibits L29-LFS synapses (Bristol et al., 2001), and because it is released in the vicinity of tail SNs and L29s, it is likely to be responsible for the facilitation of SN-MN synapses and the inhibition of L29-LFS synapses that we have observed here. Based on all of these data, we propose the following mechanistic model in a simplified neural circuit representing the T-SW reflex circuit (Fig. 10). The model is comprised 
of two neuronal cell types (tail SNs and L29 interneurons) as well as additional unidentified interneurons, which contribute to serial or parallel processing of the tactile information from the tail. In this model circuit (1) head shock induces moderate 5-HT release (Fig. 10 A, yellow modulatory field) in the pleural, pedal, and abdominal ganglia. This release is sufficient to inhibit L29LFS synapses (Bristol et al., 2001) but does not facilitate tail SN connections, thus producing overall inhibition of the T-SW reflex. (2) After contralateral tail shock, similar moderate levels of 5-HT extracellular concentrations are evoked (Fig. $10 \mathrm{~B}$, yellow fields) in the pleural, pedal, and abdominal ganglia. However, L29 interneurons fire a burst of action potentials during the stimulation, which produces post-tetanic potentiation (PTP) at their synapses with LFS siphon MNs (Frost et al., 1988). PTP at L29LFS synapses can overcome 5-HT-induced inhibition and result in overall facilitation of the synapse (Bristol et al., 2001). Surprisingly, tail SN connections also showed modest, short-lasting facilitation. The mechanisms mediating this modest facilitation are still unknown. Overall, the combined effect of such synaptic modulation would result in moderate, short-lasting sensitization of the T-SW reflex. (3) Ipsilateral tail shock releases large 5-HT concentrations in the local modulatory field surrounding tail SN cell bodies and synapses and produces robust, long-lasting facilitation of their synapses with follower interneurons (inferred by our recordings of SN-MN synapses) (Fig. 10C). This facilitated interneuronal activity is postulated to provide excitatory drive to L29 interneurons. Noxious stimulation of the tail also facilitates L29-LFS synapses (as in 2), thereby further enhancing the reflex response. Overall, these effects would result in more robust and longer-lasting sensitization of the T-SW reflex.

The T-SW reflex circuit is composed of many more neurons than tail SNs and L29 interneurons (Frost and Kandel, 1995; Barbas et al., 2003) (for review, see Cleary et al., 1995). Similarly, other nonserotonergic mechanisms probably contribute to sensitization in Aplysia (Abrams et al., 1984; Marinesco et al., 2004b). Therefore, our simplified model should not be viewed as an exhaustive description of the cellular events responsible for sensitization but rather as an illustration of the physiological role of local 5-HT modulatory fields during learning. In this regard, the fact that 5-HT release is locally regulated at multiple synaptic sites within the reflex circuit could allow the serotonergic system to generate specific spatial patterns of 5-HT release and to selectively induce short-term or long-term sensitization or behavioral inhibition of the reflex according to the noxious stimulus experienced by the animal.

Several in vitro studies have shown that Aplysia SNs can process complex patterns of 5-HT input onto their soma and/or dendritic arborization. For example, long-term synaptic facilitation can be induced if SN somata and their remote synapses are simultaneously exposed to serotonin pulses, but not if these pulses are separated by $>15 \mathrm{~min}$ (Sherff and Carew, 1999). Also, in cultured neurons, exposure of one SN synapse to multiple pulses of 5-HT produces long-term, branch-specific facilitation, which can be captured by other synaptic branches after application of a single 5-HT pulse (Martin et al., 1997). Local protein synthesis within dendrites is a key mechanism mediating such synapse-specific plasticity (Sutton and Schuman, 2005) (for review, see Martin, 2004). Our results now show that the Aplysia serotonergic system is capable of delivering such spatially restricted 5-HT pulses, thus strengthening the hypothesis that complex processing mechanisms, in some cases spatially restricted to SN somata or dendrites, can be differentially recruited in a pattern-specific manner during different forms of memory formation.

\section{References}

Abrams TW, Castelluci VF, Camardo JS, Kandel ER, Lloyd PE (1984) Two endogenous neuropeptides modulate the gill and siphon withdrawal reflex in Aplysia by presynaptic facilitation involving cAMP-dependent closure of a serotonin-sensitive potassium channel. Proc Natl Acad Sci USA 81:7956-7960.

Barbas D, DesGroseillers L, Castellucci VF, Carew TJ, Marinesco S (2003) Multiple serotonergic mechanisms contributing to sensitization in Aplysia: evidence of diverse serotonin receptor subtypes. Learn Mem 10:373-386.

Billy A, Walters E (1989) Modulation of mechanosensory threshold in Aplysia by serotonin, small cardioactive peptide B (SCPB), FMRFamide, acetylcholine and dopamine. Neurosci Lett 105:200-204.

Bristol AS, Fischer TM, Carew TJ (2001) Combined effects of intrinsic facilitation and modulatory inhibition of identified interneurons in the siphon withdrawal circuitry of Aplysia. J Neurosci 21:8990-9000.

Brunelli M, Castellucci V, Kandel ER (1976) Synaptic facilitation and behavioral sensitization in Aplysia: possible role for serotonin and cyclic AMP. Science 194:1178-1181.

Bunin MA, Wightman RM (1999) Paracrine neurotransmission in the CNS: involvement of 5-HT. Trends Neurosci 22:377-382.

Byrne JH, Kandel ER (1996) Presynaptic facilitation revisited: state and time dependence. J Neurosci 16:425-435.

Calvino MA, Iscla IR, Szczupak L (2005) Selective serotonin reuptake inhibitors induce spontaneous interneuronal activity in the leech nervous system. J Neurophysiol 93:2644-2655.

Castellucci V, Kandel E (1976) Presynaptic facilitation as a mechanism for behavioral sensitization in Aplysia. Science 194:1176-1178.

Cleary LJ, Byrne JH, Frost WN (1995) Role of interneurons in defensive withdrawal reflexes in Aplysia. Learn Mem 2:133-151.

Cleary LJ, Lee WL, Byrne JH (1998) Cellular correlates of long-term sensitization in Aplysia. J Neurosci 18:5988-5998.

Cohen JE, Onyike CU, McElroy VL, Lin AH, Abrams TW (2003) Pharmacological characterization of an adenylyl cyclase-coupled 5-HT receptor in Aplysia: comparison with mammalian 5-HT receptors. J Neurophysiol 89:1440-1455.

Erickson MT, Walters ET (1988) Differential expression of pseudoconditioning and sensitization by siphon responses in Aplysia: novel response selection after training. J Neurosci 8:3000-3010.

Fang X, Clark GA (1996) Differential responses of Aplysia siphon motor neurons and interneurons to tail and mantle stimuli: implications for behavioral response specificity. J Neurophysiol 76:3895-3909.

Fischer TM, Carew TJ (1993) Activity-dependent potentiation of recurrent inhibition: a mechanism for dynamic gain control in the siphon withdrawal reflex of Aplysia. J Neurosci 13:1302-1314.

Fitzgerald K, Carew TJ (1991) Serotonin mimics tail shock in producing transient inhibition in the siphon withdrawal reflex of Aplysia. J Neurosci 11:2510-2518

Fox L, Lloyd P (1998) Serotonergic neurons differentially modulate the efficacy of two motor neurons innervating the same muscle fibers in Aplysia. J Neurophysiol 80:647-655.

Frost WN, Kandel ER (1995) Structure of the network mediating siphonelicited siphon withdrawal in Aplysia. J Neurophysiol 73:2413-2427.

Frost WN, Clark GA, Kandel ER (1988) Parallel processing of short-term memory for sensitization in Aplysia. J Neurobiol 19:297-334.

Glanzman DL, Mackey SL, Hawkins RD, Dyke AM, Lloyd PE, Kandel ER (1989) Depletion of serotonin in the nervous system of Aplysia reduces the behavioral enhancement of gill withdrawal as well as the heterosynaptic facilitation produced by tail shock. J Neurosci 9:4200-4213.

Hawkins R, Schacher S (1989) Identified facilitator neurons L29 and L28 are excited by cutaneous stimuli used in dishabituation, sensitization, and classical conditioning of Aplysia. J Neurosci 9:4236-4245.

Hickie C, Walters ET (1995) Motor neuronal control of tail-directed and head-directed siphon responses in Aplysia californica. J Neurophysiol 74:307-321.

Illich PA, Walters ET (1997) Mechanosensory neurons innervating Aplysia siphon encode noxious stimuli and display nociceptive sensitization. J Neurosci 17:459-469.

Illich PA, Joynes RL, Walters ET (1994) Response-specific inhibition during 
general facilitation of defensive responses in Aplysia. Behav Neurosci 108:614-623.

Jacobs BL, Fornal CA (1999) Activity of serotonergic neurons in behaving animals. Neuropsychopharmacology 21:9S-15S.

Jahan-Parwar B, Rozsa KS, Salanki J, Evans ML, Carpenter DO (1987) In vivo labeling of serotonin-containing neurons by 5,7-dihydroxytryptamine in Aplysia. Brain Res 426:173-178.

Kandel ER (1979) Behavioral biology of Aplysia: a contribution to the comparative study of opisthobranch molluscs. San Francisco: Freeman.

Kupfermann I, Weiss KR (1982) Activity of an identified serotonergic neuron in free moving Aplysia correlates with behavioral arousal. Brain Res 241:334-337.

Laurienti PJ, Blankenship JE (1997) Serotonergic modulation of a voltagegated calcium current in parapodial swim muscle from Aplysia brasiliana. J Neurophysiol 77:1496-1502.

Levenson J, Byrne JH, Eskin A (1999) Levels of serotonin in the hemolymph of Aplysia are modulated by light/dark cycles and sensitization training. J Neurosci 19:8094-8103.

Lloyd PE, Kupfermann I, Weiss KR (1984) Evidence for parallel actions of a molluscan neuropeptide and serotonin in mediating arousal in Aplysia. Proc Natl Acad Sci USA 81:2934-2937.

Lotshaw DP, Lloyd PE (1990) Peptidergic and serotonergic facilitation of a neuromuscular synapse in Aplysia. Brain Res 526:81-94.

Mackey S, Carew TJ (1983) Locomotion in Aplysia: triggering by serotonin and modulation by bag cell extract. J Neurosci 3:1469-1477.

Mackey SL, Kandel ER, Hawkins RD (1989) Identified serotonergic neurons LCB1 and RCB1 in the cerebral ganglia of Aplysia produce presynaptic facilitation of siphon sensory neurons. J Neurosci 9:4227-4236.

Marinesco S, Carew TJ (2002a) Serotonin release evoked by tail-nerve stimulation in Aplysia: characterization and relationship to heterosynaptic plasticity. J Neurosci 22:2299-2312.

Marinesco S, Carew T (2002b) Improved electrochemical detection of biogenic amines in Aplysia using base-hydrolyzed cellulose-coated carbon fiber microelectrodes. J Neurosci Methods 117:87-97.

Marinesco S, Kolkman KE, Carew TJ (2004a) Serotonergic modulation in Aplysia. I. Distributed serotonergic network persistently activated by sensitizing stimuli. J Neurophysiol 92:2468-2486.

Marinesco S, Wickremasinghe N, Kolkman KE, Carew TJ (2004b) Serotonergic modulation in Aplysia. II. Cellular and behavioral consequences of increased serotonergic tone. J Neurophysiol 92:2487-2496.

Martin KC (2004) Local protein synthesis during axon guidance and synaptic plasticity. Curr Opin Neurobiol 14:305-310.

Martin KC, Casadio A, Zhu H, Yaping E, Rose JC, Chen M, Bailey CH, Kandel ER (1997) Synapse-specific, long-term facilitation of Aplysia sensory to motor synapses: a function for local protein synthesis in memory storage. Cell 91:927-938.

McPherson DR, Blankenship JE (1992) Neuronal modulation of foot and body-wall contractions in Aplysia californica. J Neurophysiol 67:23-28.

Meguid MM, Fetissov SO, Varma M, Sato T, Zhang L, Laviano A, RossiFanelli F (2000) Hypothalamic dopamine and serotonin in the regulation of food intake. Nutrition 16:843-857.
Mercer AR, Emptage NJ, Carew TJ (1991) Pharmacological dissociation of modulatory effects of serotonin in Aplysia sensory neurons. Science 254:1811-1813.

Purcell AL, Sharma SK, Bagnall MW, Sutton MA, Carew TJ (2003) Activation of a tyrosine kinase-MAPK cascade enhances the induction of longterm synaptic facilitation and long-term memory in Aplysia. Neuron 37:473-484.

Redman RS, Berry RW (1991) Temperature-dependent peptidergic feedback: potential role in seasonal egg laying in Aplysia. J Neurosci 11:1780-1785.

Rosen SC, Weiss KR, Goldstein RS, Kupfermann I (1989) The role of a modulatory neuron in feeding and satiation in Aplysia: effects of lesioning of the serotonergic metacerebral cells. J Neurosci 9:1562-1578.

Scholz KP, Byrne JH (1987) Long-term sensitization in Aplysia: biophysical correlates in tail sensory neurons. Science 235:685-687.

Sherff CM, Carew TJ (1999) Coincident induction of long-term facilitation in Aplysia: cooperativity between cell bodies and remote synapses. Science 285:1911-1914.

Siegel JM (2004) The neurotransmitters of sleep. J Clin Psychiatry 65 [Suppl 16]:4-7.

Storozhuk M, Castellucci V (1999) Modulation of cholinergic transmission in the neuronal network of the gill and siphon withdrawal reflex in Aplysia. Neuroscience 90:291-301.

Sutton MA, Schuman EM (2005) Local translational control in dendrites and its role in long-term synaptic plasticity. J Neurobiol 64:116-131.

Sutton MA, Ide J, Masters SE, Carew TJ (2002) Interaction between amount and pattern of training in the induction of intermediate- and long-term memory for sensitization in Aplysia. Learn Mem 9:29-40.

Veasey SC, Fornal CA, Metzler CW, Jacobs BL (1995) Response of serotonergic caudal raphe neurons in relation to specific motor activities in freely moving cats. J Neurosci 15:5346-5359.

Veasey SC, Fornal CA, Metzler CW, Jacobs BL (1997) Single-unit responses of serotonergic dorsal raphe neurons to specific motor challenges in freely moving cats. Neuroscience 79:161-169.

Wainwright ML, Zhang H, Byrne JH, Cleary LJ (2002) Localized neuronal outgrowth induced by long-term sensitization training in Aplysia. J Neurosci 22:4132-4141.

Wayne NL (2001) Regulation of seasonal reproduction in mollusks. J Biol Rhythms 16:391-402.

Wright W, Jones K, Sharp P, Maynard B (1995) Widespread anatomical projections of the serotonergic modulatory neuron, CB1, in Aplysia. Invert Neurosci 1:173-183.

Xin Y, Koester J, Jing J, Weiss KR, Kupfermann I (2001) Cerebralabdominal interganglionic coordinating neurons in Aplysia. J Neurophysiol 85:174-186.

Xu Y, Pieroni JP, Cleary LJ, Byrne JH (1995) Modulation of an inhibitory interneuron in the neural circuitry for the tail withdrawal reflex of Aplysia. J Neurophysiol 73:1313-1318.

Zhang H, Wainwright M, Byrne JH, Cleary LJ (2003) Quantitation of contacts among sensory, motor, and serotonergic neurons in the pedal ganglion of Aplysia. Learn Mem 10:387-393. 